\title{
The Tumor Suppressive mir-148a Is Epigenetically Inactivated in Classical Hodgkin Lymphoma
}

\author{
Julia Paczkowska ${ }^{1}$, Joanna Janiszewska ${ }^{1}{ }^{\circledR}$, Julia Bein ${ }^{2}$, Markus Schneider ${ }^{2}$, Kinga Bednarek ${ }^{1}$, \\ Adam Ustaszewski ${ }^{1}{ }^{(}$, Sylvia Hartmann ${ }^{2,3}{ }^{\circledR}$, Martin-Leo Hansmann ${ }^{2,3,4}$ and \\ Maciej Giefing 1,*(D) \\ 1 Institute of Human Genetics, Polish Academy of Sciences, 60-479 Poznan, Poland; \\ julia.paczkowska@igcz.poznan.pl (J.P.); joanna.janiszewska@igcz.poznan.pl (J.J.); \\ kinga.bednarek@igcz.poznan.pl (K.B.); adam.ustaszewski@igcz.poznan.pl (A.U.) \\ 2 Dr. Senckenberg Institute of Pathology, Goethe University Hospital, 60590 Frankfurt am Main, Germany; \\ julia.bein@kgu.de (J.B.); markus.schneider@uk-essen.de (M.S.); s.hartmann@em.uni-frankfurt.de (S.H.); \\ martin-leo.hansmann@kgu.de (M.-L.H.) \\ 3 Reference and Consultant Center for Lymph Node and Lymphoma Pathology, Goethe University, \\ 60590 Frankfurt am Main, Germany \\ 4 Frankfurt Institute for Advanced Studies, 60438 Frankfurt am Main, Germany \\ * Correspondence: maciej.giefing@igcz.poznan.pl; Tel.: +48-61-6579-138; Fax: +48-61-8233-235
}

Received: 24 September 2020; Accepted: 11 October 2020; Published: 14 October 2020

check for updates

\begin{abstract}
DNA methylation was shown previously to be a crucial mechanism responsible for transcriptional deregulation in the pathogenesis of classical Hodgkin lymphoma (cHL). To identify epigenetically inactivated miRNAs in cHL, we have analyzed the set of miRNAs downregulated in cHL cell lines using bisulfite pyrosequencing. We focused on miRNAs with promoter regions located within or $<1000 \mathrm{bp}$ from a CpG island. Most promising candidate miRNAs were further studied in primary Hodgkin and Reed-Sternberg (HRS) cells obtained by laser capture microdissection. Last, to evaluate the function of identified miRNAs, we performed a luciferase reporter assay to confirm miRNA: mRNA interactions and therefore established cHL cell lines with stable overexpression of selected miRNAs for proliferation tests. We found a significant reverse correlation between DNA methylation and expression levels of mir-339-3p, mir-148a-3p, mir-148a-5p and mir-193a-5 demonstrating epigenetic regulation of these miRNAs in cHL cell lines. Moreover, we demonstrated direct interaction between miR-148a-3p and IL15 and HOMER1 transcripts as well as between mir-148a-5p and SUB1 and SERPINH1 transcripts. Furthermore, mir-148a overexpression resulted in reduced cell proliferation in the KM-H2 cell line. In summary, we report that mir-148a is a novel tumor suppressor inactivated in $\mathrm{CHL}$ and that epigenetic silencing of miRNAs is a common phenomenon in cHL.
\end{abstract}

Keywords: cHL; epigenetic; microRNA; DNA methylation; mir-148a

\section{Introduction}

DNA methylation is a crucial mechanism responsible for deregulation of gene expression in human neoplasms. Both global DNA hypomethylation and hypermethylation of $\mathrm{CpG}$ islands, located in gene promoter regions, were widely described in tumorigenesis. Global hypomethylation increases genomic instability, whereas promoters hypermethylation result in the silencing of gene expressions [1]. However, recently new insight into the mechanisms of gene expression regulation by DNA methylation was provided. As shown for MMP-9 genes in melanoma cell lines, intragenic hypermethylation, in contrast to hypermethylation of promoter regions, positively correlates with gene expression level [2]. 
The exceptional importance of aberrant DNA methylation in the development of classical Hodgkin lymphoma (cHL) was demonstrated by several studies showing that DNA hypermethylation attenuates the expression of genes responsible for normal B-cell development [3,4]. Consequently, the neoplastic Hodgkin and Reed-Sternberg cells (HRS) of cHL show a characteristic loss of the B-cell phenotype and an increased immune escape potential [5]. Concerning the important role of DNA methylation in cHL pathogenesis, we propose that DNA methylation is co-responsible for deregulation of miRNA expression in cHL in a similar manner to protein coding genes.

The phenomenon of miRNA silencing by DNA methylation in cancer is gaining attention recently. It is known that mir-155, mir-152, mir-137, mir-31 and mir-874 expression are regulated by DNA methylation in solid tumors such as breast and prostate cancer [6,7]. Similarly, it has been shown that this mechanism contributes to miRNA downregulation in hematological malignances such as infant acute lymphoblastic leukemia or mantle cell lymphoma [8,9]. However, the significance of this process for cHL pathogenesis remains unknown and the available literature data on this phenomenon are scarce. Among the few published studies, miRNA promoter methylation and subsequent changes in microRNA expression after 5-aza-2-deoxycytidine (5-Aza-dC) treatment were described by Navarro et al. in two cHL cell lines (L-428 and L-1236) [10]. The authors have shown that altogether the expression of 13 microRNAs were induced after global DNA demethylation in both cell lines, suggesting their epigenetic inactivation.

Intrigued by these findings, we aimed to identify epigenetically inactivated miRNAs within the group of 23 downregulated miRNAs in cHL which might act as potential tumor suppressors in the disease. We decided to use unmodified cHL and non-Hodgkin lymphoma (NHL) cells lines as well as normal germinal center B-cells (GCB) from non-tumor donors in contrast to a previous study [10], to assess baseline methylation states in these malignant and normal $\mathrm{B}$ cells.

As a result of our analysis, we identified an epigenetically regulated microRNA mir-148a, not previously reported for $\mathrm{CHL}$ which could play an important role in cHL pathogenesis since it is known to be involved in the regulation of B-cell differentiation and of germinal center transcription factors [11].

\section{Materials and Methods}

\section{1. cHL and NHL Cell Lines}

Seven cHL cell lines (L-428, HDLM-2, KM-H2, L-1236, U-HO1, SUP-HD1, L-540) and 10 NHL cell lines (RAJI, DAUDI, RAMOS, NAMALWA, CA-46, VAL, OCI-LY1, OCI-LY3, OCI-LY7, SU-DHL-6) were obtained from Deutsche Sammlung von Mikroorganismen und Zellkulturen GmbH (DSMZ) or were kindly provided by Andreas Bräuninger (University Hospital Giessen, Germany) (cells: L-428, KM-H2, L-1236, L-540) [12,13]. Detailed information on cell culture conditions are presented in Table S1. Cell lines were authenticated by STR DNA profiling.

\subsection{Laser Capture Microdissection (LCM) of HRS and Non-Tumor Cells}

Frozen lymph nodes of 14 patients with cHL (10 cases: 4 nodular sclerosis, 3 mixed cellularity, 2 lymphocyte-rich and 1 undefined subset used for miRNA expression analysis and 6 cases: 3 nodular sclerosis, 2 mixed cellularity and 1 lymphocyte-rich used for DNA methylation analysis) were obtained from Dr. Senckenberg Institute of Pathology, Goethe University Hospital, Frankfurt am Main, Germany. Detailed information about clinical samples are presented in Table S2. Frozen sections $(5-10 \mu \mathrm{m})$ of the lymph nodes were mounted on membrane-covered slides (PALM, Zeiss, Bernried, Germany) and fixed in acetone. HRS cells were microdissected immediately after H\&E or CD30 immunostaining. For miRNA expression analysis approximately $1000 \mathrm{HE}$ stained cells per case were collected onto adhesive caps. For DNA methylation analysis, anti-CD30 (Clone BerH2, DAKO, Glostrup, Denmark, Super Sensitive Link-Label IHC Detection System BIOGENEX, San Ramon, CA, USA) pretreated slides were used to dissect $2 \times 200 \mathrm{HRS}$ cells and $2 \times 200$ non-tumor cells per case into $20 \mu \mathrm{L}$ PCR buffer without $\mathrm{MgCl}_{2}$ (Expand High Fidelity, Roche, Grenzach, Germany) supplemented with $0.1 \%$ 
Triton X-100. LCM was performed using the PALM laser capture microdissection microscope/system (PALM MicroBeam, Zeiss, Bernried, Germany). The study was approved by the local ethics committee of the Goethe University Hospital (157/17 from 06.04.2017).

\subsection{Sorting of $G C B C D 77^{+}$Cells}

$\mathrm{CD}^{+} \mathrm{GCB}$ cells were purified from fresh tonsils obtained from tonsillectomies of chronic hyperplastic tonsillitis using magnetic activated cell sorting (MACS; Miltenyi Biotech, Bergisch Gladbach, Germany), as described previously [14]. Informed consent was obtained from all patients according to the declaration of Helsinki. The study was approved by the local ethics committee of Goethe University Hospital (157/17 from 06.04.2017).

\subsection{DNA Isolation}

DNA isolation from cell lines was performed by phenol/chlorophorm extraction with the use of Phase Lock Gel ${ }^{\mathrm{TM}}$ tubes (5Prime Quantabio, Beverly, MA, USA) and ethanol precipitation and for MACS sorted CD77 ${ }^{+}$GCB cells by using a DNeasy Blood and Tissue Kit (Qiagen, Hilden, Germany). DNA from LCMed HRS and bystander cells for methylation analysis were obtained by cell lysis in Tris-Protein K buffer by shaking ( $600 \mathrm{rpm})$ in $55^{\circ} \mathrm{C}$ for $72 \mathrm{~h}$.

\subsection{RNA and miRNA Isolation}

Total RNA from cell lines was isolated with use of Trizol reagent based on the Chomczynski method [15]. miRNA from sorted GCB and microdissected HRS cells was isolated using miRNeasy Mini Kit (Qiagen).

\subsection{Real-Time $q P C R$}

\subsection{1. miRNA Expression Analysis}

The total RNA (10 ng) from cell lines and sorted GCB, and $10 \mu \mathrm{L}$ of the miRNA containing eluate from HRS cells were transcribed to cDNA with TaqMan ${ }^{\mathrm{TM}}$ Advanced miRNA cDNA Synthesis Kit (Applied Biosystem, Foster City, CA, USA) according to the protocol provided by the manufacturer. For reverse transcription, $3^{\prime}$ poly-A tailing and 5' adaptor sequence ligation were performed and all mature miRNAs were reverse transcribed using RT primers binding to universal sequences present on both the $5^{\prime}$ and $3^{\prime}$ extended ends. Afterward, cDNA was amplified using the Universal miR-Amp Primers and miR-Amp Master Mix. Expression of miR-148a-3p and miR-148a-5p was assessed with TaqMan ${ }^{\mathrm{TM}}$ Advanced miRNA Assays (Assay ID 477814_mir and 478718_mir) and normalized to control microRNAs: miR-361-5p and let-7g-5p (Assay ID 478056_mir and 478580_mir). The PCR reaction mix contained: $10 \mu \mathrm{L} 2 \times$ Fast Advanced Master Mix, $1 \mu \mathrm{L}$ TaqMan ${ }^{\circledR}$ Advanced miRNA Assay, $5 \mu \mathrm{L}$ of diluted cDNA template (1:10), and $4 \mu \mathrm{L} \mathrm{H}_{2} \mathrm{O}$. Reactions were run in triplicate under the following condition: $95{ }^{\circ} \mathrm{C}$ for $20 \mathrm{~s} \times 1 ;\left(95^{\circ} \mathrm{C}\right.$ for $1 \mathrm{~s}, 60^{\circ} \mathrm{C}$ for $\left.30 \mathrm{~s}\right) \times 40$.

\subsubsection{Gene Expression Analysis}

Total RNA (500 ng) from the cell lines were reverse transcribed into cDNA using the Maxima First Strand cDNA Synthesis Kit (Thermo Fisher Scientific, Waltham, MA, USA). The expression level of putative target genes for miR-148a-3p and for miR-148a-5p was evaluated in reference to the expression of ACTB and GAPDH genes. Primer sequences were designed using the Primer-BLAST software (Primer3 and BLAST) (https://www.ncbi.nlm.nih.gov/tools/primer-blast) (Table S3). The PCR reaction mix for the analyzed genes and GAPDH contained: $2 \mu \mathrm{L}$ HOT FIREPol EvaGreen qPCR Mix Plus (no Rox) (Solis Biodyne, Tartu, Estonia), $0.2 \mu \mathrm{L} F$ and R primers ( $20 \mathrm{pmo} \mu / \mu \mathrm{L}$ each), $1 \mu \mathrm{L}$ cDNA, and $6.6 \mu \mathrm{L} \mathrm{H} \mathrm{H}_{2} \mathrm{O}$. Reactions were run in triplicate under the following condition: $95^{\circ} \mathrm{C}$ for $15 \mathrm{~min} \times 1 ;\left(95^{\circ} \mathrm{C}\right.$ for $15 \mathrm{~s}, \mathrm{~T}_{\mathrm{A}}{ }^{\circ} \mathrm{C}$ for $10 \mathrm{~s}, 72{ }^{\circ} \mathrm{C}$ for $15 \mathrm{~s}$ ) $\times 40 ; 1 \times 95{ }^{\circ} \mathrm{C}$ for $30 \mathrm{~s} ; 1 \times 50{ }^{\circ} \mathrm{C}$ for $30 \mathrm{~s}$ (specific annealing temperature $\left(\mathrm{T}_{\mathrm{A}}\right)$ temperatures for all reactions are shown in Table S3). The PCR reaction mix for the 
ACTB probe consisted of: $5 \mu \mathrm{L}$ SsoAdvanced Universal Probes Supermix (Bio-Rad, Hercules, CA, USA), $0.5 \mu \mathrm{L}$ ACTB probe (Bio-Rad, Hercules, USA), $1 \mu \mathrm{L} \mathrm{cDNA}, 3.5 \mu \mathrm{L} \mathrm{H}_{2} \mathrm{O}$, and reactions were performed in triplicate under the following conditions: $95^{\circ} \mathrm{C}$ for $2 \mathrm{~min} \times 1 ;\left(95^{\circ} \mathrm{C}\right.$ for $5 \mathrm{~s}, 60^{\circ} \mathrm{C}$ for $30 \mathrm{~s}) \times 40$.

Real-time qPCR was performed using the CFX96Touch Real-Time PCR System (Bio-Rad, Hercules, USA) according to standard procedures. Results were analyzed using the Gene Expression MacroTM 1.10 software (Bio-Rad).

\section{7. mir-148a Mutation Screening}

Primer sequences for mir-148a amplification were designed using the Primer-BLAST software (https://www.ncbi.nlm.nih.gov/tools/primer-blast) (Table S3). The assay for mutation screening enclosed the genomic sequence of mir-148a with flanking regions (amplified sequence: chr7:25,989,422-25,989,721 GRCh37/hg19). The PCR reaction mix consisted of: $2 \mu \mathrm{L}$ Colorless GoTaq Flexi Buffer (Promega, Madison, WI, USA), $0.4 \mu \mathrm{L} \mathrm{MgCl}_{2}$ Solution (Promega), $0.3 \mu \mathrm{L}$ dNTP $10 \mathrm{mM}$ each (Thermo Fisher Scientific), $0.2 \mu \mathrm{L} \mathrm{F}$ and R primers ( $20 \mathrm{pmol} / \mu \mathrm{L}$ each), 0.25 GoTaq G2 Hot Start Polymerase $5 \mathrm{u} / \mu \mathrm{L}$ (Promega), and $5.65 \mu \mathrm{L} \mathrm{H}_{2} \mathrm{O}$ and $1 \mu \mathrm{L}$ DNA template $(50 \mathrm{ng} / \mu \mathrm{L})$. PCR products were obtained using the following conditions: $95{ }^{\circ} \mathrm{C}$ for $2 \mathrm{~min} \times 1 ;\left(95^{\circ} \mathrm{C}\right.$ for $30 \mathrm{~s}, 55^{\circ} \mathrm{C}$ for $30 \mathrm{~s}, 72{ }^{\circ} \mathrm{C}$ for $\left.30 \mathrm{~s}\right) \times 30$, $72{ }^{\circ} \mathrm{C}$ for $5 \mathrm{~min} \times 1$. PCR products were visualized under UV light (BioDoc-it Imaging System, UVP, Upland, USA) on 1.5\% agarose gels stained with SimplySafe (EuRx, Gdansk, Poland) and purified with ExoSap IT (Affymetrix, Santa Clara, USA). DNA sequencing reactions were performed using BigDye ${ }^{\circledR}$ Terminator v3.1 Cycle Sequencing Kit (Applied Biosystems) under the following conditions: $95^{\circ} \mathrm{C}$ for $2 \mathrm{~min} \times 1 ;\left(96^{\circ} \mathrm{C}\right.$ for $10 \mathrm{~s}, 55^{\circ} \mathrm{C}$ for $5 \mathrm{~s}, 60^{\circ} \mathrm{C}$ for $\left.240 \mathrm{~s}\right) \times 26$.

Products were purified by ethanol precipitation, separated using the ABI310 sequencer (Applied Biosystem), analyzed using the Sequencing Analysis (ABI) software, and visualized using Codon Code Aligner v.6.0.2.

\subsection{Bisulfite Pyrosequencing}

DNA bisulfite conversion was performed using EZ DNA Methylation-Gold ${ }^{\mathrm{TM}}$ Kit (Zymo Research, Irvine, CA, USA) for cell lines and GCB cells, whereas the EpiTect Plus Bisulfite Kit (Qiagen) was used for microdissected cells. The assays for bisulfite sequencing of miRNA promoter regions were designed using the PyroMark Assay Design Software 2.0.1.15 (Qiagen) (Table S3). For PCR, the PyroMark PCR kit was used to prepare the reaction mixture that contained: $12.5 \mu \mathrm{L}$ PyroMark Master Mix; $2.5 \mu \mathrm{L}$ CoralLoad; $0.5 \mu \mathrm{L}$ of $\mathrm{F}$ and R primer $(20 \mathrm{pmol} / \mu \mathrm{L}), 1 \mu \mathrm{L}$ of converted DNA $(25 \mathrm{ng} / 1 \mu \mathrm{L})$, and $8 \mu \mathrm{L} \mathrm{H}_{2} \mathrm{O}$. PCR was performed under the following conditions: $95^{\circ} \mathrm{C}$ for $15 \mathrm{~min} \times 1 ;\left(94{ }^{\circ} \mathrm{C}\right.$ for $30 \mathrm{~s}, \mathrm{~T}_{\mathrm{A}}{ }^{\circ} \mathrm{C}$ for $30 \mathrm{~s}, 72{ }^{\circ} \mathrm{C}$ for $\left.30 \mathrm{~s}\right) \times 45 ; 72{ }^{\circ} \mathrm{C}$ for $10 \mathrm{~min} \times 1 ; 4{ }^{\circ} \mathrm{C} \infty$. PCR products were visualized on $1.5 \%$ agarose gel stained with SimplySafe (EuRx) under UV light (BioDoc-it Imaging System, UVP).

Pyrosequencing was performed using the PyroMark Q24 (Qiagen) sequencer as described previously [16]. Each run included fully methylated (M-commercially available methylated DNA, Millipore, Hilden, Germany) and unmethylated controls (UMET-whole genome amplified DNA from pooled peripheral blood lymphocytes by using GenomePlex Complete Whole Genome Amplification (WGA) Kit, Sigma-Aldrich, Saint Louis, MO, USA). DNA methylation level was assessed as a mean result for all analyzed $\mathrm{CPG}$ dinucleotides for each assay. Detailed information about sequences analyzed by pyrosequencing is shown in Table S4.

For mir-148a promoter DNA methylation in microdissected cells, the same PCR conditions as described above were used, however two rounds of PCR were performed as described previously [17]. The first PCR round included: $25 \mu \mathrm{L}$ PyroMark Master Mix; $5 \mu \mathrm{L}$ CoralLoad; $1 \mu \mathrm{L}$ of F and R primer $(20 \mathrm{pmol} / \mu \mathrm{L}$ ) and $18 \mu \mathrm{L}$ of converted DNA (whole lysate). The second PCR round included a standard PCR reaction mix with $1 \mu \mathrm{L}$ of PCR product from the first round as a DNA template. 


\subsection{Dual-Luciferase Reporter Assay}

Wild type (WT) or mutant (MUT) miRNA binding sites located in the $3^{\prime}$ UTRs of selected genes were cloned into the pmirGLO Dual-Luciferase miRNA Target Expression Vector (Promega) followed by JM109 competent cells transformation (Promega). Vectors were purified using PhasePrep BAC DNA Kit (Sigma-Aldrich) and controlled by Sanger sequencing. WT and MUT oligonucleotides were designed as proposed by Mets et al. and purchased from Genomed company (Warsaw/Poland) (Table S5) [18]. In detail, WT constructs represent miRNA binding sites flanked with $30+/-$ bp of the respective 3'UTR. For MUT oligonucleotides, point mutations in miRNA binding sites were introduced in an attempt to abolish the putative interaction between the miRNA and the $3^{\prime}$ UTR.

The validation of the miRNA-3'UTR interactions was performed in the HEK 293T cells in two independent transfections and in three technical repetitions using jetPRIME DNA/siRNA (Polyplus-transfection SA, Illkirch-Graffenstaden, France) reagent as follows:

- In total, $500 \mathrm{ng}$ of vector containing the $3^{\prime} \mathrm{UTR}$ WT sequence $+50 \mu \mathrm{M}$ of the analyzed miRNA mimic (mirVana ${ }^{\circledR}$ miRNA mimic, MC10263, MC12683, Invitrogen, Carlsbad, CA, USA)

- In total, $500 \mathrm{ng}$ of vector containing the $3^{\prime} \mathrm{UTR}$ WT sequence $+50 \mu \mathrm{M}$ of the mimic negative control (mirVana ${ }^{\mathrm{TM}}$ miRNA Mimic, Negative Control \#1, Invitrogen)

- In total, $500 \mathrm{ng}$ of vector containing the $3^{\prime} \mathrm{UTR}$ MUT sequence $+50 \mu \mathrm{M}$ of the analyzed miRNA mimic (mirVana ${ }^{\circledR}$ miRNA mimic, MC10263, MC12683, Invitrogen)

- In total, $500 \mathrm{ng}$ of vector containing the $3^{\prime} \mathrm{UTR}$ MUT sequence $+50 \mu \mathrm{M}$ of the mimic negative control (mirVana ${ }^{\mathrm{TM}}$ miRNA Mimic, Negative Control \#1, Invitrogen)

Cells were lysed with Dual-Glo Luciferase Assay System $24 \mathrm{~h}$ after transfection and the bioluminescence signal of firefly luciferase was measured using the GloMax ${ }^{\circledR} 96$ Microplate Luminometer in reference to internal control of Renilla luciferase.

\subsection{0. miRNA Overexpression}

The mir-148a insert containing the $3 p$ and $5 p$ miRNA was prepared by PCR amplification using primers specific for the genomic sequence harboring the pre-miRNA-148a hairpin, including approximately 100-250 nt flanking sequence on each site, as described previously [19] (for primer sequences see Table S3). The PCR product with cohesive ends was directly cloned into the pCDH-CMV-MCS-EF1 $\alpha$-GreenPuro vector (SBI, Palo Alto, USA) and used for functional studies. The vector was packaged into lentiviral particles in HEK 293T cells using jetPRIME transfection reagent (Polyplus-transfection SA, Illkirch-Graffenstaden, France). The lentiviral particles were harvested $48 \mathrm{~h}$ after transfection and three cHL cell lines (KM-H2, L-1236 and L-540) were independently transduced by the vector carrying the mir-148a ( $3 p$ and $5 p$ ) sequence as well as by the empty vector. The vectors used contain a GFP gene and express the GFP-puromycin resistance fusion gene that enables drug selection of target cells stably expressing the microRNA. After 14 days of antibiotic selection, transduction efficiency was analyzed by flow cytometry and the overexpression of the respective miRNAs was confirmed by real-time qPCR TaqMan ${ }^{\circledR}$ Advanced miRNA Assays. Cultures showing $>75 \%$ of transduced cells were used for proliferation assays.

\subsection{Proliferation Tests}

Three transduced cHL cell lines (KM-H2, L-1236 and L-540) were seeded (500,000 cells/per well) in 24-well plates in antibiotic depleted medium after puromycin selection. The CCK8 test (Cell Counting Kit-8, Sigma-Aldrich) was performed in a time dependent manner from days 0 to 8 (measurement was performed every 2 days) to observe the differences between cells transduced with mir-148a expression and the empty vector. CCK8 assay, which is based on bio reduction of WST-8 (2-(2-methoxy-4-nitrophenyl)-3-(4-nitrophenyl)-5-(2,4-disulfophenyl)-2H-tetrazolium, monosodium salt) into formazan by cellular dehydrogenases, was performed to check the influence of 
miR-148a on cell proliferation. Cells were incubated with CCK-8 for $2 \mathrm{~h}$ in $37^{\circ} \mathrm{C}$ and the absorbance at $450 \mathrm{~nm}$ and $600 \mathrm{~nm}$ was measured using the GloMax ${ }^{\circledR} 96$ Microplate Luminometer. Experiments were performed in 4 replications in three independent reactions.

Cell proliferation was also analyzed via DNA synthesis measurement using the Click-iT Plus EdU Alexa Fluor 647 Flow Cytometry Assay Kit (Invitrogen). Cells (500,000 cells/per well) were incubated with EdU (5-ethynyl-2'-deoxyuridine) or DMSO as a control for $4 \mathrm{~h}$, fixed and permeabilized with saponin. The fluorescence signal of Alexa Fluor ${ }^{\mathrm{TM}} 647$ Click-iT $^{\mathrm{TM}}$ was detected by FlowSight ${ }^{\circledR}$ Imaging Flow Cytometer (Luminex). The experiment was performed in triplicate in two time points (days 0 and 3).

\section{Results}

\section{1. miRNA Expression in cHL Is Deregulated by DNA Hypermethylation}

Within the group of 23 miRNAs found downregulated in cHL in our parallel study (manuscript in preparation), we identified five with promoter regions located within or $<1000 \mathrm{bp}$ from a CpG island: miR-339-3p, miR-148a-3p, miR-148a-5p, miR-193a-5p, miR-4488 (Figure 1). To determine whether those miRNAs were regulated by DNA methylation, we have performed bisulfite DNA pyrosequencing for the respective promoter regions of these miRNAs in cHL cell lines $(n=7)$ and NHL cell lines $(n=10)$ as controls. We have found that the promoter region of mir-339 was hypermethylated in all cHL cell lines (range 77-89\%) and in 3 of 10 NHL cell lines (range 85-87\%), mir-148a in L-428, KM-H2, L-1236 and L-540 cell lines (range 64-91\%) and mir-193a only in L-540 (93\%). Mir-4488 was hypermethylated in 6 of 7 cHL cell lines (range 78-94\%) but also in 5 of 10 NHL cell lines (range 74-91\%) (Figure 2). For 3 of 4 analyzed miRNA promoter regions, in the case of miR-339-3p $(\mathrm{r}=-0.65, p<0.01)$, miR-148a-3p $(\mathrm{r}=-0.72, p<0.01)$, miR-148a-5p $(r=-0.74, p<0.01)$ and miR-193a-5p $(r=-0.67, p<0.01)$, their expression (based on small RNA-seq) inversely correlated with DNA methylation level (Spearman correlation).
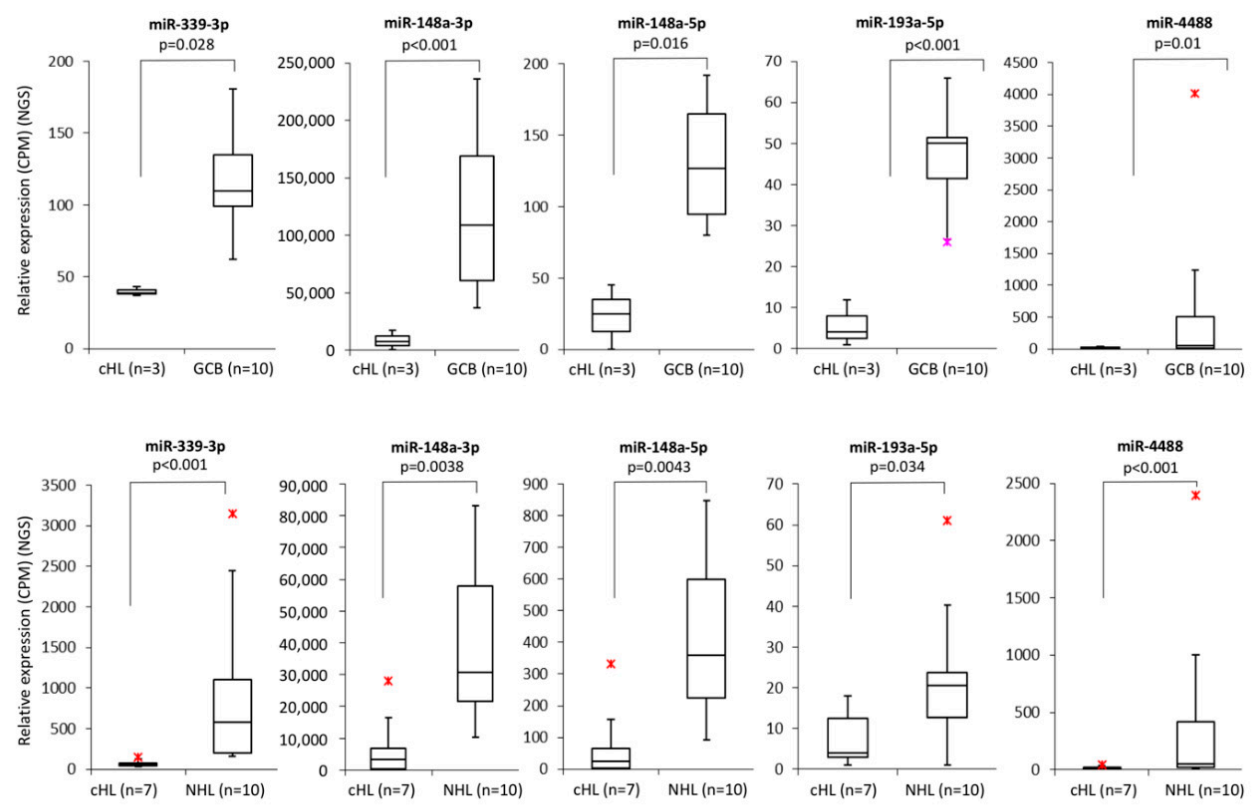

Figure 1. Expression of miRNAs (mir-339-3p, mir-148a-3p, mir-148a-5p, mir-193a-5p and mir-4488) which promoter regions are located within or up to $1000 \mathrm{bp}$ upstream from a CpG island, downregulated in cHL cell lines $(n=7)$ in comparison to NHL cell lines $(n=10)$ (based on NGS sequencing, $p<0.05$, upper panel). Expression of miRNAs (mir-339-3p, mir-148a-3p, mir-148a-5p, mir-193a-5p and mir-4488) which promoter regions are located within or up to $1000 \mathrm{bp}$ upstream from a $\mathrm{CpG}$ island, downregulated in cHL cell lines $(n=3)$ in comparison to sorted GCB $77^{+}$from tonsillectomy specimens of chronic hyperplastic tonsillitis $(n=10)$ (based on NGS sequencing, $p<0.05$, lower panel); $* / *$-min. and max. outliers. 

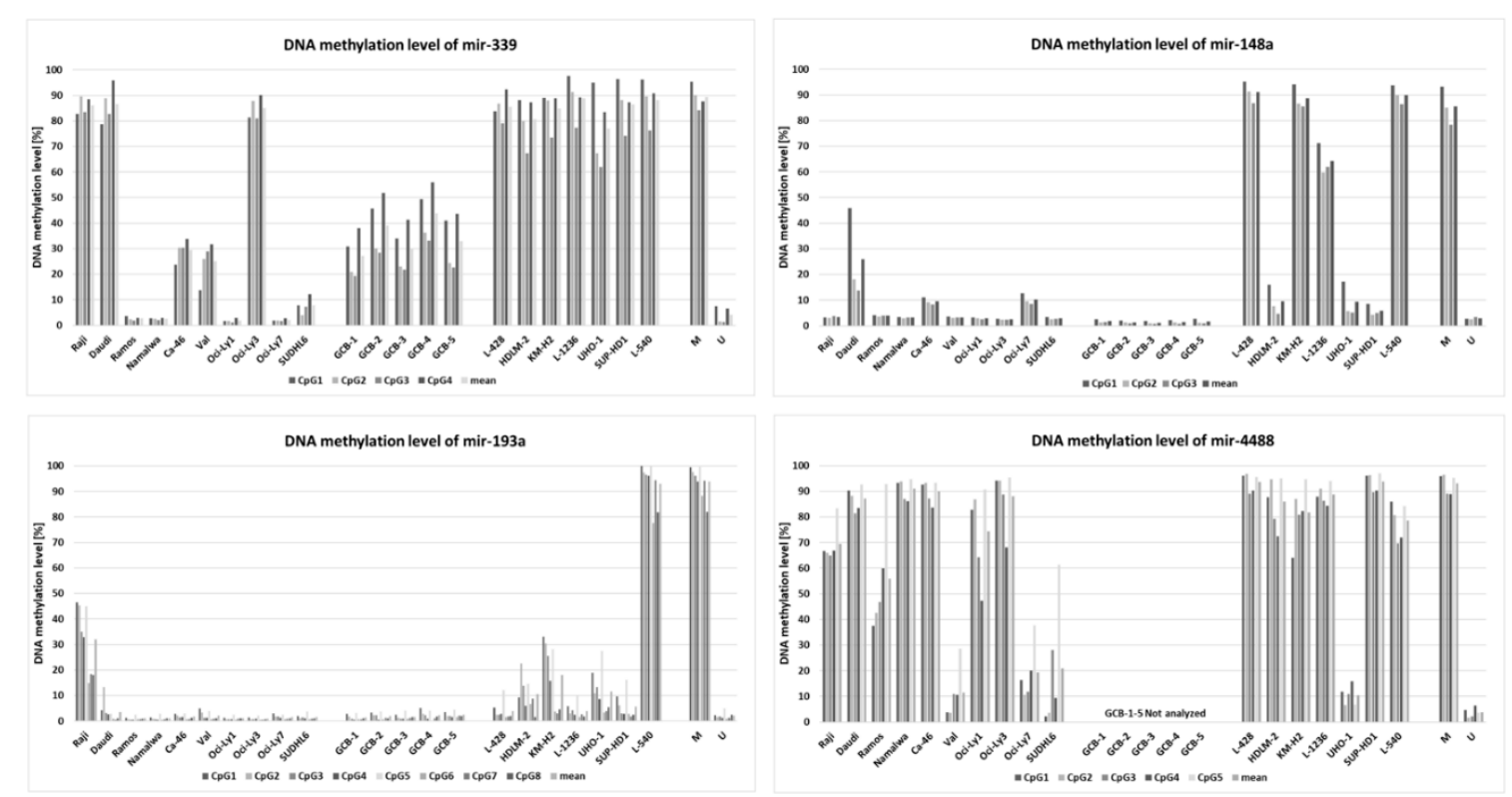

Figure 2. DNA methylation level of promoter regions of downregulated miRNAs (mir-339, mir-148a, mir-193a and mir-4488) in cHL cell lines $(n=7)$, NHL cell lines $(n=10)$ and GCB $77^{+}$from tonsillectomy specimens of chronic hyperplastic tonsillitis $(n=5)$ (except mir-4488) (analyzed by DNA bisulfite pyrosequencing).

Importantly, by further testing of these three regions (promoter of mir-339, mir-148a, mir-193a) in GCB cell pools, we observed no DNA hypermethylation for any of the chosen miRNAs (elevated DNA methylation was observed for mir-339) suggesting that DNA hypermethylation in these regions is a unique characteristic of the neoplastic cells. Because two miRNAs, namely miR-148a-3p and miR-148a-5p, were found to be recurrently silenced by DNA methylation exclusively in 4/7 cHL cell lines and not in any of the tested NHL cell lines or in GCB cells, we focused on these miRNAs in the further analysis.

Lastly, we have confirmed the downregulation of miR-148a-3p and miR-148a-5p in cHL cell lines and GCB cells using real-time qPCR with Taqman probes (Figure 3A). This shows that DNA hypermethylation downregulates miRNA gene expression and contributes to cHL-associated attenuation of miR-148a-3p and miR-148a-5p. 

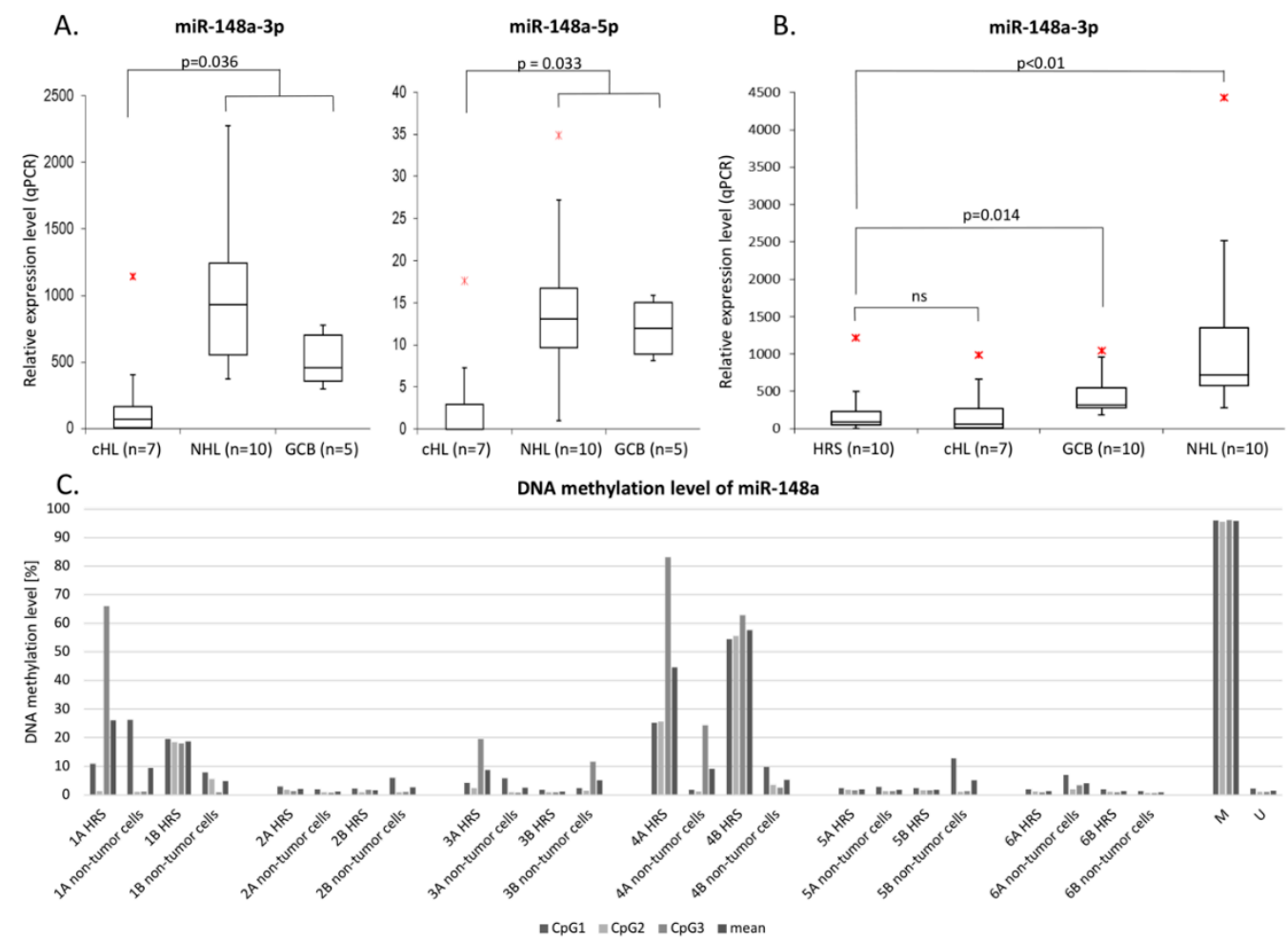

DNA methylation level of miR-148a

Figure 3. (A): Validation of mir-148a-3p/5p downregulation by real-time qPCR in cHL cell lines $(n=7)$ in comparison to NHL cell lines $(n=10)$ and GCB $77^{+}$cell pools from tonsillectomy specimens of chronic hyperplastic tonsillitis $(n=5)(p<0.05)$; $*$ - max. outlier. $(\mathbf{B})$ : miR-148a-3p downregulation in microdissected HRS cells from cHL cases $(n=10)$ in comparison to cHL cell lines $(n=7)$, NHL cell lines $(n=10)$ and GCB $77^{+}$cell pools from tonsillectomy specimens of chronic hyperplastic tonsillitis $(n=10)$ $(p<0.05)$; $*$ - max. outlier. $(\mathbf{C})$ : Elevated DNA methylation in primary microdissected HRS cells (case 1 and 4$)$ from cHL cases $(n=6)$ in comparison to non-tumor cells from the same patients.

\subsection{Canonical Gene Inactivation Mechanisms Seldomly Target mir-148a in cHL}

In order to identify further mechanisms underlying the deregulation of mir-148a in cHL, we have screened for putative copy number losses by using available results of SNP array platforms for cHL cell lines [20,21]. In two of seven evaluated cHL cell lines (L-1236, HDLM-2) with low (9\%) or moderate (64\%) mir-148a DNA methylation levels, we found heterozygous deletions that may partially explain the observed downregulation of this miRNA. In addition, we have used Sanger sequencing to identify putative mir-148a loss of function mutations. No genomic variants have been detected in the seven cHL cell lines which strengthens the hypothesis that DNA hypermethylation is the main mechanism of mir-148a deregulation.

\section{3. mir148a Is Transcriptionally Deregulated and Hypermethylated also in Primary HRS Cells}

In order to elucidate if the DNA hypermethylation and downregulation of mir-148a is not only limited to cell lines, we have performed real-time qPCR with Taqman probes in pooled HRS cells from $10 \mathrm{cHL}$ cases. Similarly to what we observed in cHL cell lines, the expression level of miR-148a-3p was significantly lower in primary HRS cells in comparison to NHL cell lines and GCB cells sorted from tonsillectomy specimens of chronic hyperplastic tonsillitis $(p<0.05)$ (Figure 3B). MiR-148a-5p expression in HRS cells was not possible to analyze due to low input of miRNA after microdissection and significantly lower endogenous expression of this miRNA in comparison to miR-148a-3p.

Lastly, we have also confirmed higher mir-148a promoter region DNA methylation levels in microdissected HRS cells from a subset of cHL primary cases by bisulfite DNA pyrosequencing. 
Two out of six evaluated cases showed elevated methylation as compared to non-tumor bystander cells from the same patient. (Figure 3C).

\subsection{IL15, HOMER1, SERPINH1 and SUB1 Are mir-148a Target Genes}

As we have shown that mir-148a ( $3 p$ and $5 p$ ) downregulation is characteristic of cHL, we were interested in identifying putative target genes that were overexpressed as a consequence of the diminished interaction with these miRNAs. We performed an in silico screen of putative target genes of these miRNAs using miRWalk 2.0 (http://zmf.umm.uni-heidelberg.de/apps/zmf/mirwalk2/index.html). We found 1421 putative target genes for miR-148a-3p, and 613 for miR-148a-5p indicated by their presence in at least 7 of 12 databases in miRWalk. By combining these results with published gene expression microarray data (Affymetryx U95) [22], we have identified 90 candidate genes for miR-148a-3p and 60 for miR-148a-5p, with at least 2-fold increased expression in cHL cell lines $(n=4$ : L-428, L-1236, KM-H2, HDLM-2) compared to normal B-cell entities ( $n=20: 5 \times$ centroblasts (CB), $5 \times$ centrocytes $(\mathrm{CC}), 5 \times$ naive B-cells, $5 \times$ memory B-cells).

For further filtering, we used the cumulative weighted context++ score $(<-0.3)$ [23] from TargetScanHuman (http://www.targetscan.org/vert_72/), a database for target prediction. This threshold indicates relatively high probability of the respective miRNA-mRNA interaction. The filtering resulted in 20 genes for miR-148a-3p and three genes for miR-148a-5p. For further analysis, we have selected top nine genes for miR-148a-3p (AKAP1, ARF4, CANX, CCT6A, C5orf30, HOMER1, IL15, FBN1, TFDP2) and three genes for miR-148a-5p (IFI6, SERPINH1, SUB1).

With the aim to validate the microarray expression data, we have performed real-time qPCR for selected genes in cHL cell lines $(n=7)$ and NHL cell lines $(n=10)$ as a control. Six candidate genes $($ HOMER1 FC $=1.93$, IL15 FC $=11.87$, FBN1 FC $=17.24$, IFI6 FC $=2.50$, SERPINH1 FC $=6.26$, SUB1 $\mathrm{FC}=1.78$ ) were overexpressed at least 1.5 -fold in cHL (Figure 4 and Supplementary Figure S1) and were further analyzed using dual-luciferase reporter assays. With this approach, we experimentally confirmed the interaction between miR-148a-3p and IL15 (40\% reduction, $p<0.01)$ and HOMER1 ( $21 \%$ reduction, $p=0.047$ ) transcripts. We have also demonstrated the interaction between miR-148a-5p and SUB1 $(22 \%$ reduction, $p<0.01)$ and SERPINH1 ( $29 \%$ reduction, $p<0.01)$ transcripts (Figure 5$).$
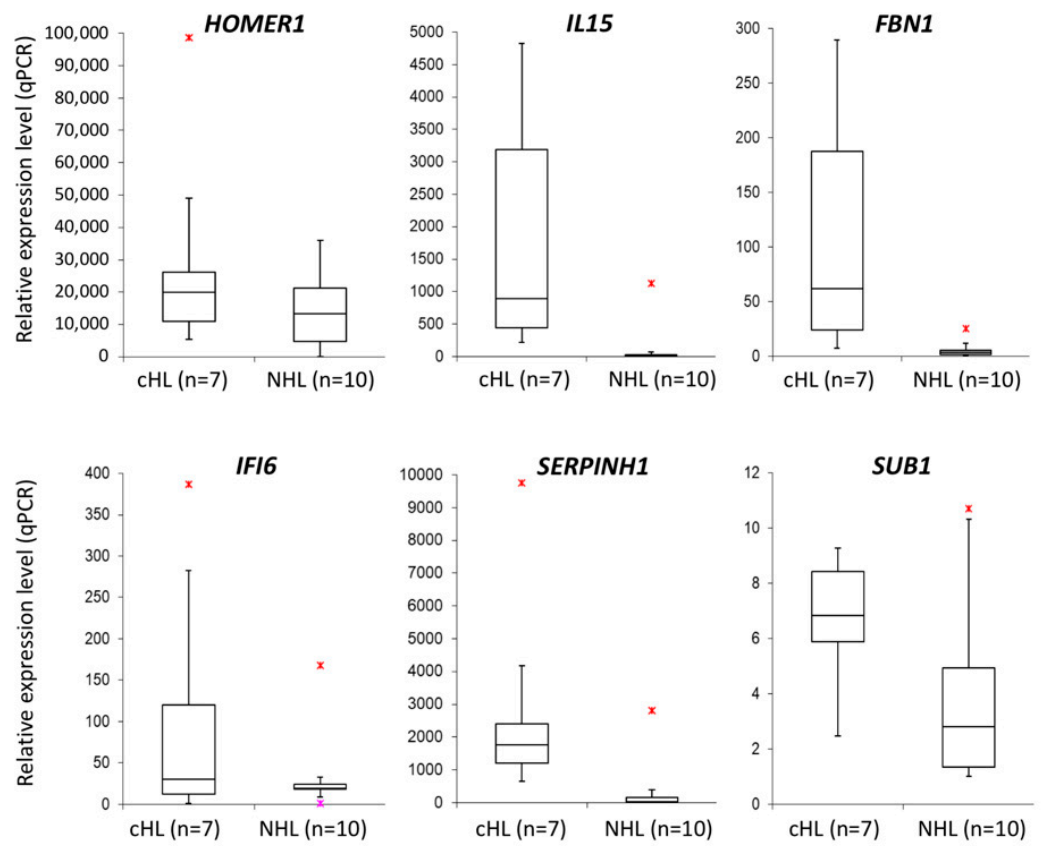

Figure 4. Overexpression of mir-148a putative target genes (HOMER1, IL15, FBN1, IFI6, SERPINH1 and SUB1) in cHL cell lines $(n=7)$ as compared to NHL cell lines $(n=10)$ (real-time qPCR); $*$ - max. outlier. 

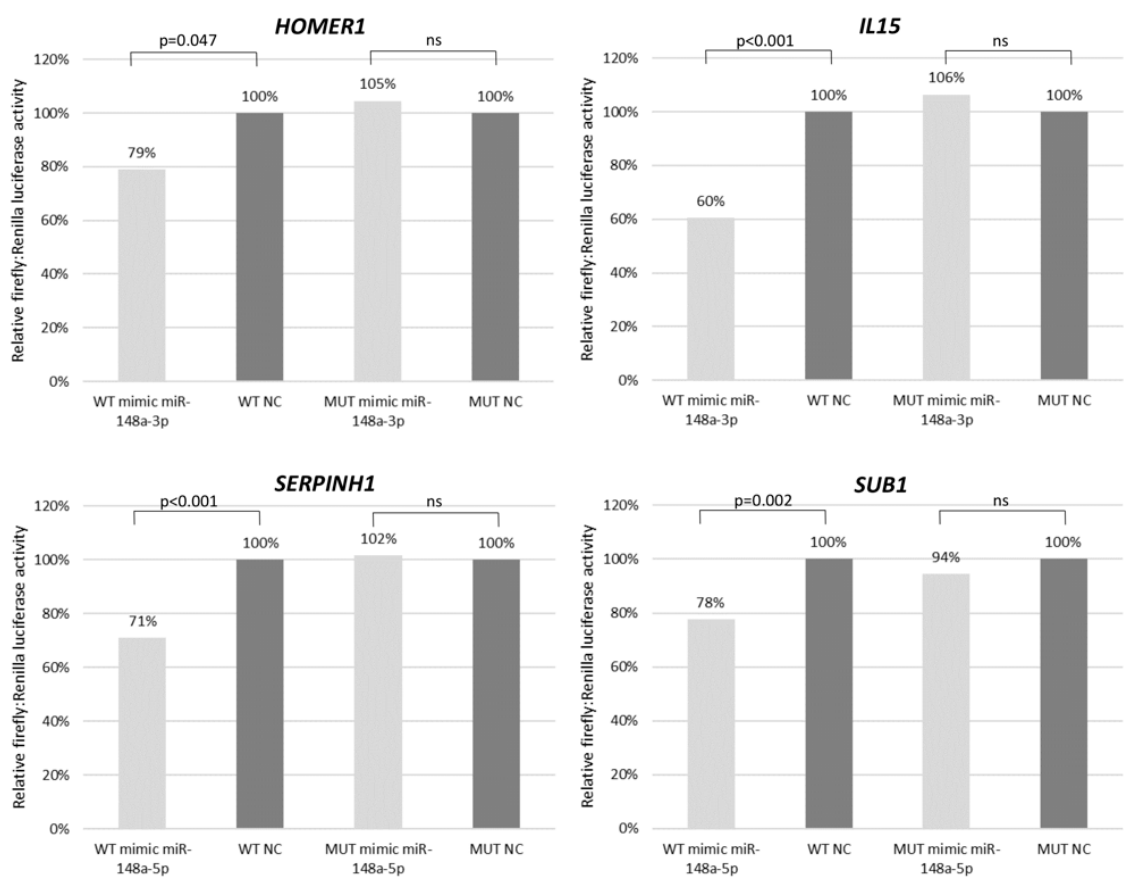

Figure 5. Interaction between mir-148a and its target genes (HOMER, IL15, SERPINH1 and SUB1 transcripts) shown by dual-luciferase reporter assay in HEK $293 \mathrm{~T}$ cell line. WT-wild type 3'UTR, MUT-mutant $3^{\prime}$ UTR with point mutations in miRNA binding site. Results presented as a mean from two independent experiment, each performed in three replications.

Based on these results, we conclude that the loss of epigenetic repression of mir-148a in cHL contributes to the deregulation of several target genes which may alter important processes in cHL pathogenesis.

\section{5. mir148a Overexpression Decreases Cell Proliferation}

In order to put the observation of mir-148a downregulation in $\mathrm{CHL}$ in a functional context, we have established three cHL cell lines stably overexpressing mir-148a (KM-H2, L-540, L-1236).

By analyzing these cell lines using the CCK8 assay we have observed a significant decrease $(p<0.05)$ in cell proliferation after miR-148a overexpression in the KM-H2 cell line. On day 8 of the experiment a $32 \%$ reduction in cell proliferation was observed in the mir-148a-expressing cell line as compared to cells with the empty vector. miR-148a overexpression had no effect on the proliferation of L-1236 and L-540 cell lines (Figure 6 and Supplementary Figures S2-S4).
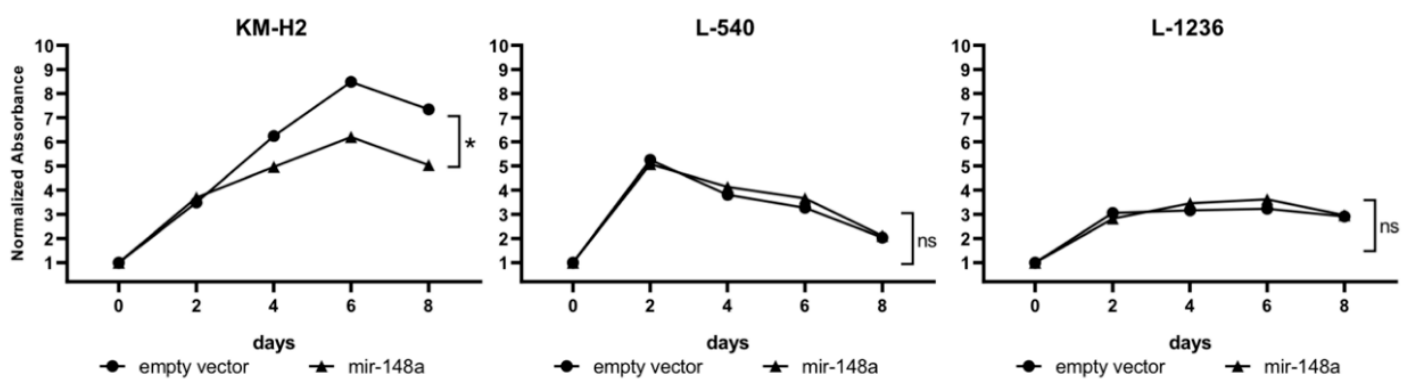

Figure 6. cHL cell lines (KM-H2, L-540, L-1236) viability after mir-148a overexpression (CCK-8 test). Results presented as a mean from three independent experiments, each performed in four replications.

To analyze if the decrease in cell proliferation observed using the CCK8 test is related to differences in DNA replication, we conducted the Click-iT ${ }^{\circledR}$ Plus EdU Alexa Fluor ${ }^{\circledR} 647$ Flow Cytometry Assay 
based on measurement of newly synthesized DNA in cells in S-phase. In line with the CCK8 test results, a decrease in DNA synthesis was observed for the KM-H2 cell line. On day 3 of culture, KM-H2 cells transduced with miR-148a expression vector showed a significantly lower percentage of cells with newly synthesized DNA as compared to cells transduced with the empty vector ( $35 \%$ vs. $49 \%$, $p=0.03$ ).

Taken together, functional studies performed in cell lines with induced mir-148a overexpression indicate that this microRNA has a negative influence on proliferation and DNA synthesis in a subset of cHL cell lines. Therefore, we assume that mir-148a may act as a tumor suppressor at least in some cHL cases.

\section{Discussion}

DNA methylation was previously shown to be an essential mechanism responsible for regulation of gene expression in the pathogenesis of $\mathrm{cHL}[3,4]$. Our findings suggest that epigenetic silencing in $\mathrm{cHL}$ is not limited to protein coding genes but also plays an important role in deregulation of miRNA expression. miRNAs are responsible for fine-tuning the expression of protein coding genes involved in the maturation of B-cells, therefore deregulation of miRNA expression might contribute to the development of B-cell lymphomas [24,25].

As cHL is defined by a unique miRNA expression profile distinct from other B-cell lymphomas, one can expect the presence/absence of driver miRNAs having significant influence on cHL pathogenesis $[26,27]$. Following this lead, we have analyzed whether miRNAs found downregulated in cHL cell lines in our parallel study (our unpublished results, manuscript in preparation) have their promoter region located within or $<1000 \mathrm{bp}$ from a CpG island. We assumed that this is a strong indication that these miRNAs are epigenetically regulated and their DNA methylation level should be evaluated.

Consistent with our hypothesis, we have found promoter region hypermethylation of mir-339, mir-148a, mir-193a and mir-4488 in cHL cell lines. Moreover, with the exception of mir-4488, we have demonstrated that DNA methylation level inversely correlates with the expression level of these miRNAs. Importantly, mir-148a was found epigenetically attenuated exclusively in cHL.

Mir-148a was previously reported to be involved in the development of other type of cancers such as stomach, liver, lung, and breast cancer [28]. There are several ways in which it can contribute to cHL pathogenesis, however reports on its involvement in cHL are lacking so far. Firstly, mir-148a has been described as a component of a regulatory circuit that involves the NF- $\mathrm{kB}$ pathway, which is activated in HRS cells [29]. In this model, epigenetically mediated downregulation of mir-148a results in the overexpression of NF-kB in cancer cells. Secondly, by the interaction with methyltransferase DNMT3b and DNMT1, miR-148a-3p are directly involved in the process of DNA methylation, which is essential in the context of the global deregulation of methylation machinery in cHL [30,31]. Thirdly, mir-148a is expressed during physiological B-cell activation, and the promotor region of mir-148a is rich in motifs recognized by B-cell specific transcription factors like ELF1, EBF1, and E2A [9]. This is in line with our observation that this miRNA is unmethylated and expressed in GCB cells. Therefore, downregulation of mir-148a in cHL will likely contribute to the deregulation of the normal B-cell maturation process.

However, the exact function of most of the mir-148a target genes validated in our study in cHL remain poorly understood. Only the role of IL15, a prominent pro-inflammatory cytokine and important component of the growth and survival signals in cHL was previously described [32]. In the study by Ullrich et al., IL15 stimulation of cHL cell lines resulted in increased proliferation and activation of MAP kinase and JAK/STAT5 pathway. Interestingly, HOMER1 expression is also regulated via MAPK pathways and has a potential anti-apoptotic function [33]. SERPINH1 and SUB1 in turn were described as oncogenes in different cancer types promoting cell proliferation and invasion [34]. Exogenous overexpression of SUB1 in nude mice was shown to lead to transformation of normal multipotent fibroblast and tumorigenesis [35]. Taken together, the observed downregulation of mir-148a-3p/5p 
may lead to loss of transcriptional control of several cancer-related genes and at least partially explain their overexpression in cHL.

Lastly, in an attempt to understand the biological effect of the observed downregulation of mir-148a-3p/5p in cHL cell lines and microdissected HRS cells, we established three cHL cell lines (KM-H2, L-1236 and L-540) with stable mir-148a overexpression. Functional assays revealed the involvement of these miRNAs in negative regulation of proliferation in the KM-H2 cell line. We can only speculate that the composition of genetic alterations in the KM-H2 cell line makes it more sensitive to mir-148a overexpression than in the L-1236 and L-540 cell lines.

In summary, we identify mir-148a as a novel tumor-suppressive miRNA that is epigenetically inactivated in cHL.

\section{Conclusions}

We propose that miRNAs undergo epigenetic silencing by DNA hypermethylation in cHL in the same way as protein coding genes. Moreover, we identified mir-148a to be silenced by recurrent DNA hypermethylation which leads to loss of transcriptional control over several target genes including IL15 and HOMER1 and thereby contribute to cHL pathogenesis.

Supplementary Materials: The following are available online at http://www.mdpi.com/2073-4409/9/10/2292/s1, Table S1: Cell culture conditions, Table S2: The clinical data of patients, Table S3: Primer sequences used in the study, Table S4: Assays used for bisulfite pyrosequencing (genomic localization based on GRCh37/hg19), Table S5: Oligonucleotide sequences used in luciferase assay. Figure S1: Expression of putative mir-148a target genes: HOMER1, IL15, FBN1, IFI6, SERPINH1 and SUB1 in seven cHL cell lines. Figure S2: KM-H2 viability after mir-148a overexpression (CCK-8 test). Results presented separately for each of the biological repetitions. Figure S3: L-540 viability after mir-148a overexpression (CCK-8 test). Results presented separately for each of the biological repetitions. Figure S4: L-1236 viability after mir-148a overexpression (CCK-8 test). Results presented separately for each of the biological repetitions.

Author Contributions: J.P.-Designed the study, performed experiments, analyzed data, prepared figures and tables, wrote the manuscript; J.J.-Performed experiments and analyzed data; J.B.-Performed experiments; K.B., A.U.-Analyzed data; M.S., S.H.--Interpreted data, characterized and selected cases; M.-L.H.-Characterized and selected cases; M.G.-Co-designed the study, supervised the research and data analyses, wrote the manuscript. All authors have read and agreed to the published version of the manuscript.

Funding: This study was supported by the Polish National Science Center grant: 2014/14/M/NZ2/00529 to M.G. J.P. was supported by Etiuda scholarship of the Polish National Science Center UMO-2019/32/T/NZ5/00441.

Acknowledgments: The authors would like to thank the Molecular Genetics Laboratory in Poznan (Laboratorium Genetyki Molekularnej) for performing cell lines authentication and Nicole Cullen for language editing.

Conflicts of Interest: The authors declare no conflict of interest.

\section{References}

1. Pfeifer, G. Defining Driver DNA Methylation Changes in Human Cancer. Int. J. Mol. Sci. 2018, $19,1166$. [CrossRef] [PubMed]

2. Falzone, L.; Salemi, R.; Travali, S.; Scalisi, A.; McCubrey, J.A.; Candido, S.; Libra, M. MMP-9 overexpression is associated with intragenic hypermethylation of MMP9 gene in melanoma. Aging (Albany NY) 2016, 8, 933-940. [CrossRef] [PubMed]

3. Ushmorov, A.; Leithäuser, F.; Sakk, O.; Weinhaüsel, A.; Popov, S.W.; Möller, P.; Wirth, T. Epigenetic processes play a major role in B-cell-specific gene silencing in classical Hodgkin lymphoma. Blood 2006, 107, $2493-2500$. [CrossRef] [PubMed]

4. Ammerpohl, O.; Haake, A.; Pellissery, S.; Giefing, M.; Richter, J.; Balint, B.; Kulis, M.; Le, J.; Bibikova, M.; Drexler, H.G.; et al. Array-based DNA methylation analysis in classical Hodgkin lymphoma reveals new insights into the mechanisms underlying silencing of B cell-specific genes. Leukemia 2012, 26, 185-188. [CrossRef]

5. Schwering, I.; Bräuninger, A.; Klein, U.; Jungnickel, B.; Tinguely, M.; Diehl, V.; Hansmann, M.L.; Dalla-Favera, R.; Rajewsky, K.; Küppers, R. Loss of the B-lineage-specific gene expression program in Hodgkin and Reed-Sternberg cells of Hodgkin lymphoma. Blood 2003, 101, 1505-1512. [CrossRef] [PubMed] 
6. Daniunaite, K.; Dubikaityte, M.; Gibas, P.; Bakavicius, A.; Rimantas Lazutka, J.; Ulys, A.; Jankevicius, F.; Jarmalaite, S. Clinical significance of miRNA host gene promoter methylation in prostate cancer. Hum. Mol. Genet. 2017, 26, 2451-2461. [CrossRef]

7. Zhang, L.; Yan, D.L.; Yang, F.; Wang, D.D.; Chen, X.; Wu, J.Z.; Tang, J.H.; Xia, W.J. DNA methylation mediated silencing of microRNA-874 is a promising diagnosis and prognostic marker in breast cancer. Oncotarget 2017, 8, 45496-45505. [CrossRef]

8. Nishi, M.; Eguchi-Ishimae, M.; Wu, Z.; Gao, W.; Iwabuki, H.; Kawakami, S.; Tauchi, H.; Inukai, T.; Sugita, K.; Hamasaki, Y.; et al. Suppression of the let-7b microRNA pathway by DNA hypermethylation in infant acute lymphoblastic leukemia with MLL gene rearrangements. Leukemia 2013, 27, 389-397. [CrossRef]

9. Yim, R.L.; Wong, K.Y.; Kwong, Y.L.; Loong, F.; Leung, C.Y.; Chu, R.; Lam, W.W.; Hui, P.K.; Lai, R.; Chim, C.S. Methylation of miR-155-3p in mantle cell lymphoma and other non-Hodgkin's lymphomas. Oncotarget 2014, 5, 9770-9782. [CrossRef]

10. Navarro, A.; Díaz, T.; Cordeiro, A.; Díaz Beyá, M.; Ferrer, G.; Fuster, D.; Martinez, A.; Monzó, M. Epigenetic regulation of microRNA expression in Hodgkin lymphoma. Leuk. Lymphoma 2015, 56, 2683-2689. [CrossRef]

11. Porstner, M.; Winkelmann, R.; Daum, P.; Schmid, J.; Pracht, K.; Côrte-Real, J.; Schreiber, S.; Haftmann, C.; Brandl, A.; Mashreghi, M.F.; et al. miR-148a promotes plasma cell differentiation and targets the germinal center transcription factors Mitf and Bach2. Eur. J. Immunol. 2015, 45, 1206-1215. [CrossRef] [PubMed]

12. Drexler, H.; MacLeod, R.; Nagel, S.; Dirks, W.; Uphoff, C.; Steube, K.; Quentmeier, H. Guide to leukemia-lymphoma cell lines on CD. Blood 2005, 106, 4340. [CrossRef]

13. Mader, A.; Bruderlein, S.; Wegener, S.; Melzner, I.; Popov, S.; Muller-Hermelink, H.K.; Barth, T.F.; Viardot, A.; Moller, P. U-HO1, a new cell line derived from a primary refractory classical Hodgkin lymphoma. Cytogenet. Genome Res. 2007, 119, 204-210. [CrossRef]

14. Brune, V.; Tiacci, E.; Pfeil, I.; Döring, C.; Eckerle, S.; van Noesel, C.J.; Klapper, W.; Falini, B.; von Heydebreck, A.; Metzler, D.; et al. Origin and Pathogenesis of Nodular Lymphocyte Predominant Hodgkin Lymphoma as Revealed by Global Gene Expression Analysis. J. Exp. Med. 2008, 205, 2251-2268. [CrossRef]

15. Chomczynski, P. A reagent for the single-step simultaneous isolation of RNA, DNA and proteins from cell and tissue samples. Biotechniques 1993, 15, 532-534.

16. Szaumkessel, M.; Richter, J.; Giefing, M.; Jarmuz, M.; Kiwerska, K.; Tönnies, H.; Grenman, R.; Heidemann, S.; Szyfter, K.; Siebert, R. Pyrosequencing-based DNA methylation profiling of Fanconi anemia/BRCA pathway genes in laryngeal squamous cell carcinoma. Int. J. Oncol. 2011, 39, 505-514. [CrossRef] [PubMed]

17. Schneider, M.; Szaumkessel, M.; Richter, J.; Ammerpohl, O.; Hansmann, M.L.; Küppers, R.; Siebert, R.; Giefing, M. The PRDX2 gene is transcriptionally silenced and de novo methylated in Hodgkin and Reed-Sternberg cells of classical Hodgkin lymphoma. Blood 2014, 123, 3672-3674. [CrossRef]

18. Mets, E.; Van Peer, G.; Van der Meulen, J.; Boice, M.; Taghon, T.; Goossens, S.; Mestdagh, P.; Benoit, Y.; De Moerloose, B.; Van Roy, N.; et al. MicroRNA-128-3p is a novel oncomiR targeting PHF6 in T-cell acute lymphoblastic leukemia. Haematologica 2014, 99, 1326-1333. [CrossRef] [PubMed]

19. Kluiver, J.; Slezak-Prochazka, I.; van den Berg, A. Studying microRNAs in lymphoma. Methods Mol. Biol. 2013, 971, 265-276. [PubMed]

20. Schmidt, A.; Schmitz, R.; Giefing, M.; Martin-Subero, J.I.; Gesk, S.; Vater, I.; Massow, A.; Maggio, E.; Schneider, M.; Hansmann, M.L.; et al. Rare occurrence of biallelic CYLD gene mutations in classical Hodgkin lymphoma. Genes Chromosomes Cancer 2010, 49, 803-809. [CrossRef] [PubMed]

21. Otto, C.; Giefing, M.; Massow, A.; Vater, I.; Gesk, S.; Schlesner, M.; Richter, J.; Klapper, W.; Hansmann, M.L.; Siebert, R.; et al. Genetic lesions of the TRAF3 and MAP3K14 genes in classical Hodgkin lymphoma. Br. J. Haematol. 2012, 157, 702-708. [CrossRef] [PubMed]

22. Küppers, R.; Klein, U.; Schwering, I.; Distler, V.; Bräuninger, A.; Cattoretti, G.; Tu, Y.; Stolovitzky, G.A.; Califano, A.; Hansmann, M.L.; et al. Identification of Hodgkin and Reed-Sternberg cell-specific genes by gene expression profiling. J. Clin. Investig. 2003, 111, 529-537. [CrossRef] [PubMed]

23. Agarwal, V.; Bell, G.; Nam, J.; Bartel, D. Predicting effective microRNA target sites in mammalian mRNAs. Elife 2015, 4, e05005. [CrossRef] [PubMed]

24. Sole, C.; Larrea, E.; Manterola, L.; Goicoechea, I.; Armesto, M.; Arestin, M.; Caffarel, M.; Araujo, A.; Fernandez, M.; Araiz, M.; et al. Aberrant expression of MicroRNAs in B-cell lymphomas. Microrna 2016, 5, 87-105. [CrossRef] 
25. Solé, C.; Larrea, E.; Di Pinto, G.; Tellaetxe, M.; Lawrie, C.H. miRNAs in B-cell lymphoma: Molecular mechanisms and biomarker potential. Cancer Lett 2017, 405, 79-89. [CrossRef]

26. Cordeiro, A.; Monzó, M.; Navarro, A. Non-Coding RNAs in Hodgkin Lymphoma. Int. J. Mol. Sci. 2017, 18, 1154. [CrossRef]

27. Yuan, Y.; Kluiver, J.; Koerts, J.; de Jong, D.; Rutgers, B.; Abdul Razak, F.R.; Terpstra, M.; Plaat, B.E.; Nolte, I.M.; Diepstra, A.; et al. miR-24-3p Is Overexpressed in Hodgkin Lymphoma and Protects Hodgkin and Reed-Sternberg Cells from Apoptosis. Am. J. Pathol. 2017, 187, 1343-1355, Erratum in 2019, $189,479$. [CrossRef]

28. Li, Y.; Deng, X.; Zeng, X.; Peng, X. The Role of Mir-148a in Cancer. J. Cancer 2016, 7, 1233-1241. [CrossRef]

29. Xu, Q.; Liu, L.Z.; Yin, Y.; He, J.; Li, Q.; Qian, X.; You, Y.; Lu, Z.; Peiper, S.C.; Shu, Y.; et al. Regulatory circuit of PKM2/NF-KB/miR-148a/152-modulated tumor angiogenesis and cancer progression. Oncogene 2015, 34, 5482-5493. [CrossRef]

30. Zhan, Q.; Fang, Y.; Deng, X.; Chen, H.; Jin, J.; Lu, X.; Peng, C.; Li, H.; Shen, B. The Interplay Between miR-148a and DNMT1 Might be Exploited for Pancreatic Cancer Therapy. Cancer Investig. 2015, 33, 267-275. [CrossRef]

31. Duursma, A.M.; Kedde, M.; Schrier, M.; le Sage, C.; Agami, R. miR-148 targets human DNMT3b protein coding region. RNA 2008, 14, 872-877. [CrossRef] [PubMed]

32. Ullrich, K.; Blumenthal-Barby, F.; Lamprecht, B.; Köchert, K.; Lenze, D.; Hummel, M.; Mathas, S.; Dörken, B.; Janz, M. The IL-15 cytokine system provides growth and survival signals in Hodgkin lymphoma and enhances the inflammatory phenotype of HRS cells. Leukemia 2015, 29, 1213-1218. [CrossRef]

33. Luo, P.; Zhao, Y.; Li, D.; Chen, T.; Li, S.; Chao, X.; Liu, W.; Zhang, L.; Qu, Y.; Jiang, X.; et al. Protective effect of Homer 1a on tumor necrosis factor- $\alpha$ with cycloheximide-induced apoptosis is mediated by mitogen-activated protein kinase pathways. Apoptosis 2012, 17, 975-988. [CrossRef] [PubMed]

34. Chakravarthi, B.V.; Goswami, M.T.; Pathi, S.S.; Robinson, A.D.; Cieślik, M.; Chandrashekar, D.S.; Agarwal, S.; Siddiqui, J.; Daignault, S.; Carskadon, S.L.; et al. MicroRNA-101 regulated transcriptional modulator SUB1 plays a role in prostate cancer. Oncogene 2016, 35, 6330-6340. [CrossRef] [PubMed]

35. Shi, C.; Zhu, Y.; Chung, L.W.K.; Su, Y.; Cheng, T. PC4 is a novel oncogenic gene for mesenchymal stem cell transformation and mediates the reciprocal actions between mesenchymal stem cells and prostate cancer cells. Exp. Hematol. 2008, 36, 82-83.

Publisher's Note: MDPI stays neutral with regard to jurisdictional claims in published maps and institutional affiliations.

(C) 2020 by the authors. Licensee MDPI, Basel, Switzerland. This article is an open access article distributed under the terms and conditions of the Creative Commons Attribution (CC BY) license (http://creativecommons.org/licenses/by/4.0/). 